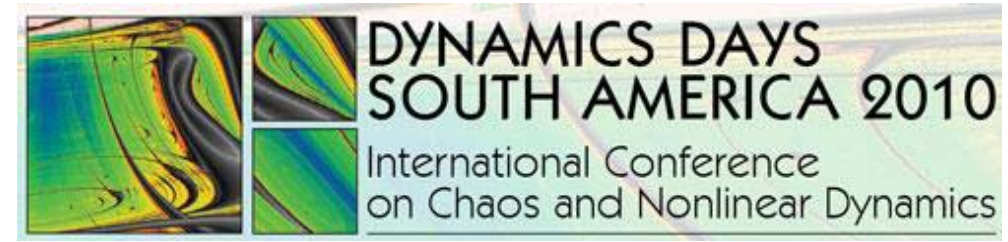

INPE - National Institute for Space Research

São José dos Campos - SP - Brazil - July 26-30, 2010

\title{
NONLINEAR ELECTRONIC TRANSPORT BEHAVIOR IN INDIUM NITRIDE
}

\author{
Clóves G. Rodrigues ${ }^{I}$, Áurea R. Vasconcellos ${ }^{2}$, R. Luzzi $^{2}$ \\ ${ }^{1}$ Departamento de Física, PUC-GO, CP 86, 74605-010 Goiânia-GO, Brazil, cloves@pucgoias.edu.br \\ ${ }^{2}$ Instituto de Física, UNICAMP, 13083-970 Campinas-SP, Brazil, rlgmesd@ifi.unicamp.br
}

keywords: Applications of Nonlinear Sciences; Applications in Engineering and Nanoscience.

In recent decades the III-Nitrides semiconductors (like Gallium Nitride, Aluminium Nitride, and Indium Nitride) have been the object of intensive study. This is a consequence of the realization of the long-awaited possibility of growing satisfactory samples, leading to the increased development of electronic and opto-electronic devices (blue diodes, blue lasers, etc.) of large technological and industrial interest $[1,2,3]$. The GaN and AlN are semiconductors with band gaps and other characteristics that largely differ from those of InN (it may be noticed that it resembles the case of InAs in the family of the III-V semiconductors). Recently, the band gap of InN has been clarified as being $0.64 \mathrm{eV}$ [4-8], which corresponds to the near infra-red region and enables AlN-GaN-InN-based nitride materials to be used for fabricating devices operating from the UV to near infra-red range. These properties of nitride semiconductors are being used to develop high efficiency tandem-type solar cells.

Further, InN can be readily made as the n-type by introducing crystal defects [3]. Of large relevance are the studies of their optical and transport properties in situations in which they are working in far-fromequilibrium conditions. When under the action of intermediate to strong electric fields their transport properties are nonlinear (in the electric field intensity). We have considered such question in a series of papers (e.g. Refs. [9-10]), and in the present paper we are adding a study on the coupled influence of the electric field strength and the concentration of carriers on the nonlinear mobility (that is, a mobility dependent nonlinearly on field and concentration) in n-doped InN.

In the study of transport phenomena it has been used variations of computational modeling approaches (in the domain of Nonequilibrium Molecular Dynamics and related to Monte Carlo computational methods). On the other hand, improved analytical methods, that is, nonlinear quantum kinetic theories for studying physical phenomena in systems arbitrarily departed from equilibrium, are desirable. An advantage that they may present in relation to the computational-modeling methods resides in that the explicit equation of motion for the basic nonequilibrium thermodynamic variables, which characterize the dissipative macrostate of the system, provide a better physical insight and interpretation of the results.

Nonequilibrium quantum kinetic theories based on various intuitive techniques and ideas are presently available. One particularly appropriate for the purpose just stated, and which offers an elegant and concise analytical treatment of the theory of irreversible processes, has been derived from an approach based on a nonequilibrium ensemble formalism (since it can be founded on a variational principle, consisting in the maximization of the informational entropy in the statistical description, for short it is referred to as MaxEnt-NESOM [17-23]). We call the attention to the fact that it can be considered a farreaching generalization of Mori's formalism [17-23]. It is applied in next section to the study of nonlinear transport properties in nonequilibrium semiconductors in order to derive the basic set of equations of evolution for the chosen basic variables which describe the nonequilibrium (irreversible) thermodynamic macrostate of the system, with numerical calculations for $\mathrm{InN}$ presented in section III. The electric current and the mobility in the steady state are obtained, and their dependence on the electric field strength (in this nonlinear domain) and on the concentration is obtained and analyzed.

We consider a n-doped direct-gap polar semiconductor (for numerical calculations we use the parameters corresponding to wurtzite $\mathrm{InN}$ ), in conditions such that the extra electrons provided by the impurities act as mobile carriers in the conduction band (i.e. they are on the metallic side of Mott transition). We use the effectivemass approximation which requires that in explicit applications it needs be set an upper limit value for the electric field, fixed by the condition that for smaller values than this one intervalley scattering can be neglected and the parabolic band approximation is satisfactory.

The Hamiltonian of the system is composed of the energies of the free electrons and phonons, the interaction of the electrons with the impurities, the electron-phonon interaction, the anharmonic interaction of the $l o$ phonons involving $a c$ phonons, the interaction of the electrons with the electric field, and the interaction of the acoustic phonons with the external thermal reservoir, and it is assumed a good thermal contact such that the $a c$ phonons remain in near equilibrium at the reservoir temperature, and we recall that the to phonons practically do not interact with the conduction band electrons.

To characterized the nonequilibrium thermodynamic 
state of the system, considering that the presence of the electric field changes the energy of the electrons (they acquire energy in excess of equilibrium), and that they keep transferring this excess to the, mainly, lo phonons (via Fröhlich interaction), and that an electric current (flux of electrons) is produced.

We solve numerically the set of coupled nonlinear integro-differential equations to obtain the time evolution of the basic intensive nonequilibrium thermodynamic variables for wurtzite $\mathrm{n}$-InN. The scattering mechanisms considered are: 1) polar optical phonon, 2) piezoelectric, 3 ) acoustic deformation potential, and 4) ionized impurity [33-41]. We assumed that all donors are ionized and that the free electron concentration is equal to the dopant concentration. The electric field applied to $\mathrm{InN}$ is restricted to be smaller than $20 \mathrm{kV} / \mathrm{cm}$ since intervalley scattering is not considered in this work.

We observed that $\mathrm{InN}$ was initially reported to have an energy gap of 1.8 to $2.0 \mathrm{eV}$ [11], but since 2001, values from $0.67 \mathrm{eV}$ (low-temperature) to $0.63 \mathrm{eV}$ (roomtemperature) have been reported based on the optical properties of high-quality hexagonal $\mathrm{InN}$ epitaxial layers with low background electron densities grown by molecular beam epitaxy (MBE) [3-5]. In addition, while initial experimental results suggested an electron effective mass of $0.11 \mathrm{~m}_{0}$ [12], more recent experimental measurements have instead suggested an electron effective mass of $0.04 \mathrm{~m}_{0}[3,8]$ for this material (here $\mathrm{m}_{0}$ denotes the free electron mass).

Finally, summarizing the results thus obtained, we can say that the mobility is weakly dependent on the concentrations up to $\sim 5 \times 10^{17} \mathrm{~cm}^{-3}$. For larger concentrations it keeps decreasing with an increasing negative slope, particularly for concentrations $n>2 x$ $10^{18} \mathrm{~cm}^{-3}$; this behavior can be characterized as a kind of transition between two regimes. The existence of these two regimes is a consequence of a similar behavior in the dependence of the nonequilibrium temperatures on the concentration, namely, $\mathrm{T}_{\mathrm{e}}$ and $\mathrm{T}_{\mathrm{LO}}$ (nonequilibrium temperatures of electrons and lo phonons, respectively) are nearly constant for $n<5 \times 10^{17} \mathrm{~cm}^{-3}$ and both increase (with an increasing slope) for $n>2 \times 10^{18} \mathrm{~cm}^{-3}$. Moreover, with increasing thermal bath temperature the mobility keeps decreasing with a tendency to be near equal for all the field intensities considered for values of $\mathrm{T}_{0}$ roughly above twice the room temperature.

Acknowledgments: This work was supported by State of Goiás Research Agency (FAPEG), São Paulo State Research Agency (FAPESP), and Brazilian Research Council (CNPq).

\section{References}

[1] J. Piprek, (ed.), Nitride Semiconductor Devices: Principles and Simulation, Wiley, New York, USA, 2007.

[2] H. Morkoç, Handbook of Nitride Semiconductors and Devices, Wiley, New York, USA, 2008.

[3] K. Takahashi, A. Yoshikawa, A. Sandhu, (eds.) "Wide Bandgap Semiconductors", Springer, Berlin, Germany, 2006.

[4] J. Wu, W. Walukiewicz, W. Shan, K.M. Yu, J.W. Ager, S.X. Li, E.E. Haller, H. Lu, W.J. Schaff, J. Appl. Phys., Vol. 94, p. 4457, 2003.

[5] Y. Ishitani, H. Masuyama, W. Terashima, M. Yoshitani, N. Hashimoto, S.B. Che, A. Yoshikawa, Phys. Status Solid C, Vol. 2, p. 2276, 2005.

[6] V.Y. Davydov, A.A. Klochikhin, V.V. Emtsev, S.V. Ivanov, V.V. Vekshin, F. Bechstedt, J. Furthmüller, H. Harima, A.V. Mudryi, A. Hashimoto, A. Yamamoto, J. Aderhold, J. Graul, E.E. Haller, Phys. Status Solidi B, Vol. 230, p. R4, 2002.

[7] T. Matsuoka, H. Okamoto, M. Nakao, H. Harima, E. Kurimoto, Appl. Phys. Lett., Vol. 81, p. 1246, 2002.

[8] J. Wu, W. Walukiewicz, K.M. Yu, J.W. Ager III, E.E. Haller, Hai Lu, W.J. Schaff, Y. Saito, Y. Nanishi, Appl. Phys. Lett., Vol. 80, p. 3967, 2002.

[9] C.G. Rodrigues, A.R. Vasconcellos, R. Luzzi, "Theoretical calculations of nonlinear electronic transport behavior in III-nitrides: GaN and AlN", Phys. Status Solidi (b), Vol. 246, No. 2, p. 417, 2009.

[10] C.G. Rodrigues, A.R. Vasconcellos, R. Luzzi, "Optical properties of III-nitrides in electric fields", Eur. Phys. J. B, Vol. 72, No. 1, p. 67, 2009.

[11] T.L. Tansley, C.P. Foley, J. Appl. Phys., Vol. 59, p. 3241, 1986.

[12] Y.C. Yeo, T.C. Chong, M.F. Li, J. Appl. Phys., Vol. 83, p. 1429, 1998. 